\title{
Against all odds: blended phenotypes of three single-gene defects
}

\author{
Yong $\mathrm{Li}^{1,4}$, Anika Salfelder ${ }^{2,4}$, Karl Otfried Schwab ${ }^{2}$, Sarah Catharina Grünert ${ }^{2}$, Tanja Velten ${ }^{2}$, \\ Dieter Lütjohann ${ }^{3}$, Pablo Villavicencio-Lorini ${ }^{2,5}$, Uta Matysiak-Scholze ${ }^{2}$, Bernhard Zabel ${ }^{2}$, \\ Anna Köttgen ${ }^{\star, 1}$ and Ekkehart Lausch ${ }^{\star, 2}$
}

Whole-exome sequencing allows for an unbiased and comprehensive mutation screening. Although successfully used to facilitate the diagnosis of single-gene disorders, the genetic cause(s) of a substantial proportion of presumed monogenic diseases remain to be identified. We used whole-exome sequencing to examine offspring from a consanguineous marriage featuring a novel combination of congenital hypothyroidism, hypomagnesemia and hypercholesterolemia. Rather than identifying one causative variant, we report the first instance in which three independent autosomal-recessive single-gene disorders were identified in one patient. Together, the causal variants give rise to a blended and seemingly novel phenotype: we experimentally characterized a novel splice variant in the thyroglobulin gene (c.638+5G $>A$ ), resulting in skipping of exon 5 , and detected a pathogenic splice variant in the magnesium transporter gene TRPM6 (c.2667+1G $>$ A), causing familial hypomagnesemia. Based on the third variant, a stop variant in ABCG5 (p.(Arg446*)), we established a diagnosis of sitosterolemia, confirmed by elevated blood plant sterol levels and successfully initiated targeted lipid-lowering treatment. We propose that blended phenotypes resulting from several concomitant single-gene disorders in the same patient likely account for a proportion of presumed monogenic disorders of currently unknown cause and contribute to variable genotype-phenotype correlations.

European Journal of Human Genetics (2016) 24, 1274-1279; doi:10.1038/ejhg.2015.285; published online 27 January 2016

\section{INTRODUCTION}

Rare monogenic disorders are often diagnosed late, primarily due to missing medical awareness and knowledge. Morbidity and mortality are commonly worsened by a protracted diagnosis. Although causal therapies are not available for most single-gene disorders, prevention of complications and avoidance of ineffective and/or contraindicated medical interventions can dramatically improve individual outcomes. ${ }^{1}$

Despite tremendous progress in genomic medicine over the last decade, the underlying genetic defect has only been identified for approximately one-third to one-half of the 7000-15000 presumed monogenic diseases. ${ }^{2,3}$ Purported reasons for the large proportion of unresolved monogenic diseases include complicated genetic architecture, technical limitations, variants of unclear pathogenicity and/or occurring de novo, and incomplete or incorrect phenotyping. An additional explanation that has so far received little attention, is that a proportion of the unresolved monogenic diseases in fact may represent 'blended phenotypes' ${ }^{2,5}$ that arise from the presence of molecular causes of more than one monogenic disease in an individual. This may be especially relevant for offspring from consanguineous matings. ${ }^{6}$ Here, we describe the first case of a patient in whom a combination of three recessive single-gene disorders gives rise to a clinical picture of congenital hypothyroidism, hypomagnesemia and hypercholesterolemia.

\section{MATERIALS AND METHODS}

Patients and materials

Patients were recruited by physician-initiated referral. The study was conducted in accordance with the Declaration of Helsinki protocols and approved by the institutional ethics review board of Freiburg University Hospital, Germany. Written informed consent for molecular studies was obtained from the affected individuals and/or their legal guardians in accordance with current German law (GenDG). Control samples and primary cells were collected from ancestry-, sex- and age-matched healthy individuals under the same criteria and regulations. DNA and RNA were obtained from peripheral-blood leukocytes by standard extraction procedures. ${ }^{7}$

\section{Whole-exome sequencing data analysis}

Exome sequences were enriched from DNA using the Agilent SureSelect Exome Enrichment kit V4 (Agilent Technologies, Waldbronn, Germany). Sequencing was performed on the 5500xl SOLiD System (Life Technologies, Darmstadt, Germany); 75 bp single-end reads were produced. Sequence alignment and variant calling were performed using the targeted re-sequencing workflow in LifeScope v2.5.1 (Life Technologies). The reads were aligned to the human hg19 reference genome. Variant calling stringency was set to high. Called variants were annotated and filtered using GeneTalk (http://www.gene-talk.de/). ${ }^{8}$ For the purpose of variant filtering, HomozygosityMapper (http://www.homozygositymapper.org//) $)^{9}$ was used to detect regions of homozygosity shared by the two patients but not their mother. In addition, regions of homozygosity were detected individually in the two patients using HomozygosityMapper. When

${ }^{1}$ Renal Division, Department of Internal Medicine, Medical Center, University of Freiburg, Freiburg, Germany; ${ }^{2}$ Center for Pediatrics and Adolescent Medicine, Medical Center, University of Freiburg, Freiburg, Germany; ${ }^{3}$ Institute of Clinical Chemistry and Clinical Pharmacology, University Clinics of Bonn, Germany

*Correspondence: Dr A Köttgen, Department of Internal Medicine, University Medical Center Freiburg, Freiburg 79106, Germany. Tel: +49 761 270 78050 ; E-mail: anna. koettgen@uniklinik-freiburg.de

or Dr E Lausch, Department of Pediatrics, University Medical Center Freiburg, Freiburg 79106, Germany. Tel: +49 76127043630 ; Fax: +49 761 270 44710; E-mail: ekkehart. lausch@uniklinik-freiburg.de

${ }^{4}$ These authors contributed equally to this work.

${ }_{5}^{5}$ Present address: Institute of Human Genetics, Martin Luther University Halle-Wittenberg, Germany.

Received 31 August 2015; revised 21 November 2015; accepted 16 December 2015; published online 27 January 2016 
filtering for autosomal-recessive candidate variants, we retained stop, splice, frameshift and non-synonymous variants with prevalence $<1 \%$ in the 1000 Genomes Project and Exome Sequencing Project. Filtered candidate variants mapping into regions of homozygosity were prioritized, but all other filtered candidate variants were also examined for potentially causal variants. Details on additional bioinformatics analyses, including those of publicly available RNAseq data from thyroid tissue to characterize the effects of the variant underlying hypothyroidism, are available in the Supplementary Material.

\section{Variant confirmation and molecular studies}

For the genomic Sanger and cDNA analysis, exons including intron-exon boundaries of TRPM6 (NM_017662.4; exon numbering according to NG_017036.1; variant affects splice donor site adjacent to exon 20), ABCG5 (NM_022436.2; exon numbering according to NG_008883.1; variant affects exon 10), and TG (NM_003235.4; exon numbering according to NG_015832.1; variant affects splice donor site adjacent to exon 5) were PCR-amplified using standard protocols as described previously; ${ }^{7}$ details are given in the Supplementary Material. The three variants and phenotypes have been submitted to ClinVar database (accession numbers SCV000257394 (TRPM6), SCV000257395 (ABCG5), SCV257396 (TG)).

\section{Measurement of blood sterols}

Cholesterol, its metabolite $5 \alpha$-cholestanol, the plant sterols campesterol, stigmasterol and sitosterol as well as their $5 \alpha$-saturated metabolites $5 \alpha$ campestanol and $5 \alpha$-sitostanol were determined by gas chromatography-flame ionization detection using 5 a-cholestane as internal standard. ${ }^{10}$ Structure identity was verified by gas chromatography-mass spectrometry in Scan Modus comparing the spectra with those from authentic highly pure compounds.

\section{RESULTS}

The index patients are a 19-year-old boy (individual II-01) and his 14-year-old sister (individual II-02) from a family of Turkish origin (compare with pedigree in Figure 2). Their parents are first-degree cousins; both paternal and maternal grandparents were also reportedly related. Both siblings were diagnosed with congenital primary hypothyroidism by TSH newborn screening within the first 3 days of life and given thyroid hormone daily since. In postnatal examination and follow-up, hypothyroidism was classified as a non-goiterous thyroid developmental disorder of unknown etiology. Laboratory parameters and sonography on regular follow-up visits indicated thyroid dysgenesis and secretory thyroid hormone insufficiency with an orthotopic, but small and dysplastic thyroid (Figure 1a, Supplementary Figure S1A). Normal audiometry in both children made Pendred Syndrome (MIM \#274600) unlikely; no further investigations to classify primary hypothyroidism were undertaken. In the following years, the children had no developmental problems under adapted thyroxine substitution.

However, at the age of 2 years, the girl was brought to the emergency room with generalized seizures. Ictal EEG showed generalized spike or polyspikes-and-wave complexes (Supplementary Figure S1B). The fit responded well to emergent benzodiazepine treatment. Later, clinical presentation and EEG changes were consistent with a diagnosis of myoclonic-astatic epilepsy (MAE). MAE, also known as Doose Syndrome (MIM \#607208), is an epilepsy syndrome of early childhood usually characterized by difficult to control generalized seizures. Laboratory results at that time were unremarkable and she was started on anticonvulsant levetiracetam. Although no additional a
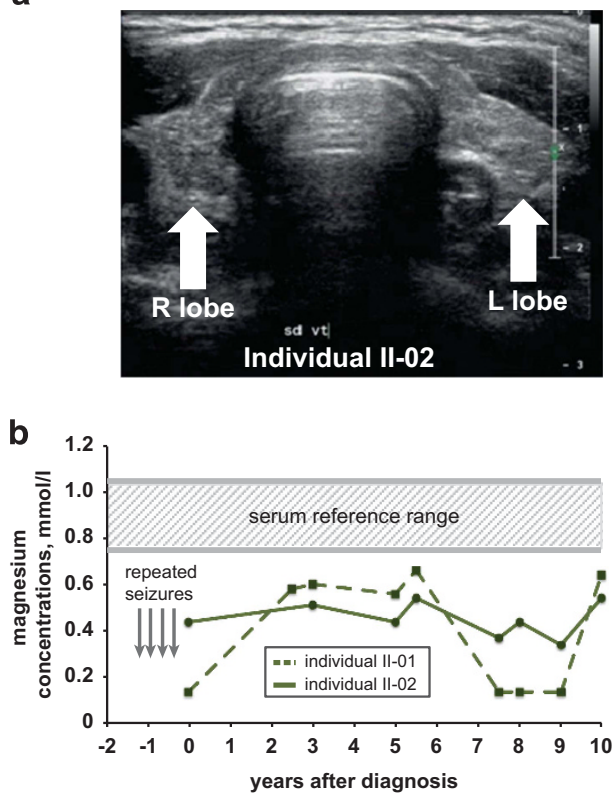

C

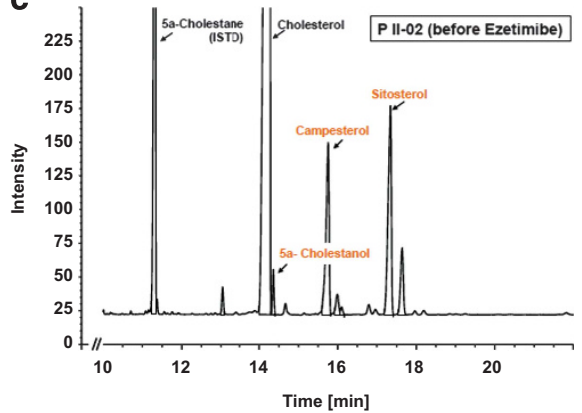

d
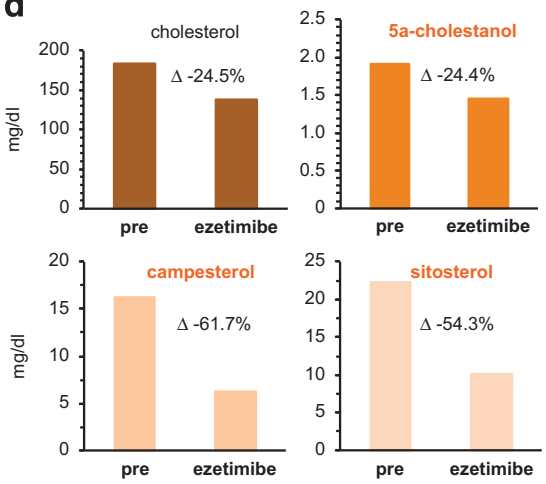

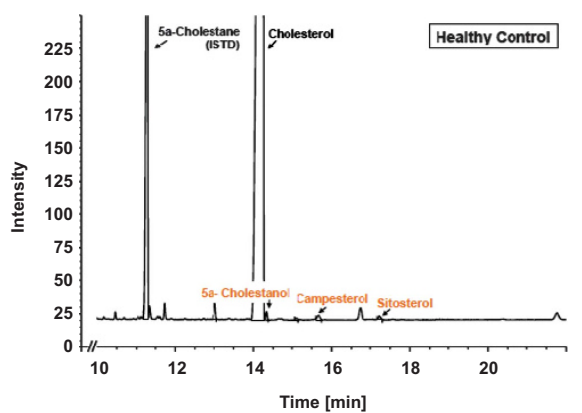

Figure 1 (a) Representative transverse thyroid sonogram of the girl (individual II-02) at the age of 13 years. Volume of both lobes was reduced and echogenicity increased, but no cystic lesions were found. Measurement of the thyroid gland was performed using a Philips ultrasound machine with a $7.5 \mathrm{MHz}$ transducer. For each thyroid lobe, the maximum perpendicular anteroposterior (depth) and mediolateral (width) diameters were measured on a transverse image of the largest diameter. The greatest craniocaudal (length) diameter of each lobe was measured on a longitudinal image. (b) Time course of serum magnesium levels in both index patients. Dashed lines/squares represent individual II-01 and solid lines/circles individual II-02. Arrows indicate the time range when repeated seizures occurred in the girl but do not represent the number of episodes. Reference range for serum magnesium: 0.75-1.05 mmol/I (gray area). (c) Gas chromatographic (GC) separation combined with flame ionization detection (FID) of the trimethylsilyl ethers of cholesterol, $5 \alpha$-cholestanol, campesterol, and sitosterol from a serum sample of the patient (PII-02) shortly before the start of ezetimibe treatment, in comparison with extracted sterols from an healthy, age- and gender-matched Control subject. (d) Treatment with 10 mg ezetemibe for 13 months significantly reduced cholesterol as well as plant sterols (values based on one measurement at each time point). 
generalized seizure occurred over several months, she continued to suffer from several myoclonic events per week. On a subsequent blood draw, severe hypomagnesemia with $<0.2 \mathrm{mmol} / \mathrm{l}$ was unraveled (Figure 1b, Supplementary Table S1). Serum magnesium levels had not been checked in any previous investigation. The other electrolytes, $\mathrm{pH}$, and bicarbonate levels had always been normal. Anticonvulsants were discontinued and switched to oral magnesium substitution therapy, which proved effective to cure MAE instantly. Furthermore, EEG changes completely normalized even though blood magnesium levels remained subnormal (Figure 1c). Testing in the brother revealed low magnesium levels as well, although he had never been symptomatic (Figure 1c, Table 1). No changes were found in his EEG and ECG investigations. Electrolytes were normal in both parents. Further work-up of hypomagnesemia excluded Gitelman Syndrome (MIM \#263800), and both siblings received a tentative diagnosis of defective intestinal resorption/loss of magnesium.

On a routine blood draw, hypercholesterolemia was diagnosed in the girl (Table 1). As concomitant TSH was suppressed and fT3 and fT4 values were normal, hypercholesterolemia was not secondary to hypothyroidism. In spite of a low-normal BMI and no dietary particularities, both her LDL-cholesterol and total cholesterol levels were increased, while triglycerides were normal (type IIA, Table 1). No clinical signs of systemic cholesterol deposition evolved, and evaluation of intima-media thickness by high-resolution B-mode echoDoppler sonography demonstrated normal findings for her age at several occasions. An attempt to treat hyperlipidemia by cholestyramine at the age of 9 years lowered cholesterol levels initially but high cholesterol levels were observed on several occasions thereafter.
Notwithstanding, under thyroid hormone replacement therapy and oral substitution of magnesium, both patients developed well and attended school without problems.

Given that both siblings from this consanguineous marriage were affected, we suspected a potential genetic origin. As no known monogenic disorder matched our patients' multi-faceted phenotype, we assumed an underlying recessive disease of yet unknown origin. Therefore, whole-exome sequencing (WES) of the two index patients, their parents and paternal grandparents was undertaken to search for causal variants.

High-quality WES data were obtained from the two patients, their mother, and the paternal grandparents. Detailed summary statistics are provided in Supplementary Table S1. Subsequently, variant annotation and filtering gave rise to a short list of 73 candidate variants. Although the attempts to pinpoint a single variant that could explain the observed clinical phenotype were unsuccessful, we identified separate variants in three genes known to cause single-gene disorders that collectively explained the clinical phenotype.

\section{A splice variant in TRPM6 causes hypomagnesemia}

Regions of homozygosity comprised $5.7 \%$ of the boy's genome (166437.6 kb, hg19) and $7.2 \%$ of the girl's genome $(207969.7 \mathrm{~kb})$. Focusing on the $95476.1 \mathrm{~kb}$ regions of homozygosity shared by the children but not their mother (Supplementary Figure S2), we identified the variant c. $2667+1 \mathrm{G}>\mathrm{A}$ in the donor splice site adjacent to exon 20 of TRPM6, for which both patients were homozygous (Supplementary Table S2). TRPM6 encodes a magnesium transport protein expressed predominantly in intestinal and renal epithelia,

Table 1 Laboratory results of the two patients

\begin{tabular}{|c|c|c|c|}
\hline Parameter & Age at measurement boy/girl (reference range ${ }^{a}$ ) & Individual II-01 (boy) & Individual II-02 (girl) \\
\hline \multicolumn{4}{|l|}{ Thyroid function } \\
\hline TSH & Day 3/day 2 (<39 mU/l) & $>50 \mathrm{mU} / \mathrm{l}$ & $>50 \mathrm{mU} / \mathrm{l}$ \\
\hline fT4 & Day 3/day 2 (26-63 pmol/l) & $20 \mathrm{pmol} / \mathrm{l}$ & $18 \mathrm{pmol} / \mathrm{l}$ \\
\hline TG & Day 3/day $2(1.4-78 \mathrm{ng} / \mathrm{ml})$ & $1 \mathrm{ng} / \mathrm{ml}$ & $0.8 \mathrm{ng} / \mathrm{ml}$ \\
\hline$\alpha-\mathrm{TG}$ & 12 years $/ 14$ years $(<64 \mathrm{IU} / \mathrm{ml})$ & 20.6 & $20 \mathrm{IU} / \mathrm{ml}$ \\
\hline$\alpha$-TPO & 12 years/14 years $(<26 \mathrm{IU} / \mathrm{ml})$ & 13.8 & $9.4 \mathrm{IU} / \mathrm{ml}$ \\
\hline \multicolumn{4}{|l|}{ Electrolytes } \\
\hline Mg (serum) & 6 years $/ 2$ years $(0.7-0.91 \mathrm{mmol} / \mathrm{l})$ & $<0.2 \mathrm{mmol} / \mathrm{l}$ & $<0.2 \mathrm{mmol} / \mathrm{l}$ \\
\hline Mg (urine) & 6 years $/ 2$ years $(1.2-1.7 \mathrm{mmol} / \mathrm{l})$ & $<0.2 \mathrm{mmol} / \mathrm{l}$ & $<0.2 \mathrm{mmol} / \mathrm{l}$ \\
\hline Mg (urine excretion) & 7 years/3 years $(3-5 \mathrm{mmol} / 24 \mathrm{~h})$ & $1.5 \mathrm{mmol} / 24 \mathrm{~h}$ & $0.8 \mathrm{mmol} / 24 \mathrm{~h}$ \\
\hline Ca (serum) & 6 years/2 years $(2.0-2.7 \mathrm{mmol} / \mathrm{l})$ & $1.8 \mathrm{mmol} / \mathrm{l}$ & $2.03 \mathrm{mmol} / /$ \\
\hline \multicolumn{4}{|l|}{ Lipids } \\
\hline Cholesterol & 10 years/6 years $(120-240 \mathrm{mg} / \mathrm{dl})$ & $113 \mathrm{mg} / \mathrm{dl}$ & $300 \mathrm{mg} / \mathrm{dl}$ \\
\hline LDL-cholesterol & 6 years $(50-155 \mathrm{mg} / \mathrm{dl})$ & ND & $219 \mathrm{mg} / \mathrm{dl}$ \\
\hline VLDL-cholesterol & 6 years $(<30 \mathrm{mg} / \mathrm{dl})$ & ND & 30 mg/dl \\
\hline HDL-cholesterol & 6 years $(45-100 \mathrm{mg} / \mathrm{dl})$ & ND & 54 mg/dl \\
\hline Triglycerides & 10 years/6 years $(<150 \mathrm{mg} / \mathrm{dl})$ & 99 mg/dl & $106 \mathrm{mg} / \mathrm{dl}$ \\
\hline Cholesterol (GC/MS) & 13 years $(120-240 \mathrm{mg} / \mathrm{dl})$ & ND & $170 \mathrm{mg} / \mathrm{dl}$ \\
\hline \multicolumn{4}{|l|}{ Plant sterols } \\
\hline Cholestanol (GC/MS) & 13 years (NA) & ND & $2.01 \mathrm{mg} / \mathrm{dl}$ \\
\hline Lathosterol (GC/MS) & 13 years (NA) & ND & $0.108 \mathrm{mg} / \mathrm{dl}$ \\
\hline Campesterol (GC/MS) & 13 years (NA) & ND & $16.5 \mathrm{mg} / \mathrm{dl}$ \\
\hline Sitosterol (GC/MS) & 13 years (NA) & ND & $20.2 \mathrm{mg} / \mathrm{dl}$ \\
\hline
\end{tabular}

Abbreviations: HDL, high-density lipoprotein; LDL, low-density lipoprotein; GC/MS, gas chromatography-mass spectrometry; ND, not determined; NA, not available; TG, triglycerides; TSH, thyroid stimulating hormone; VLDL, very-low-density lipoprotein.

aPediatric reference values of the Institute of Clinical Chemistry and Laboratory Medicine of Freiburg University.

Values outside the reference range are marked by bold script. 

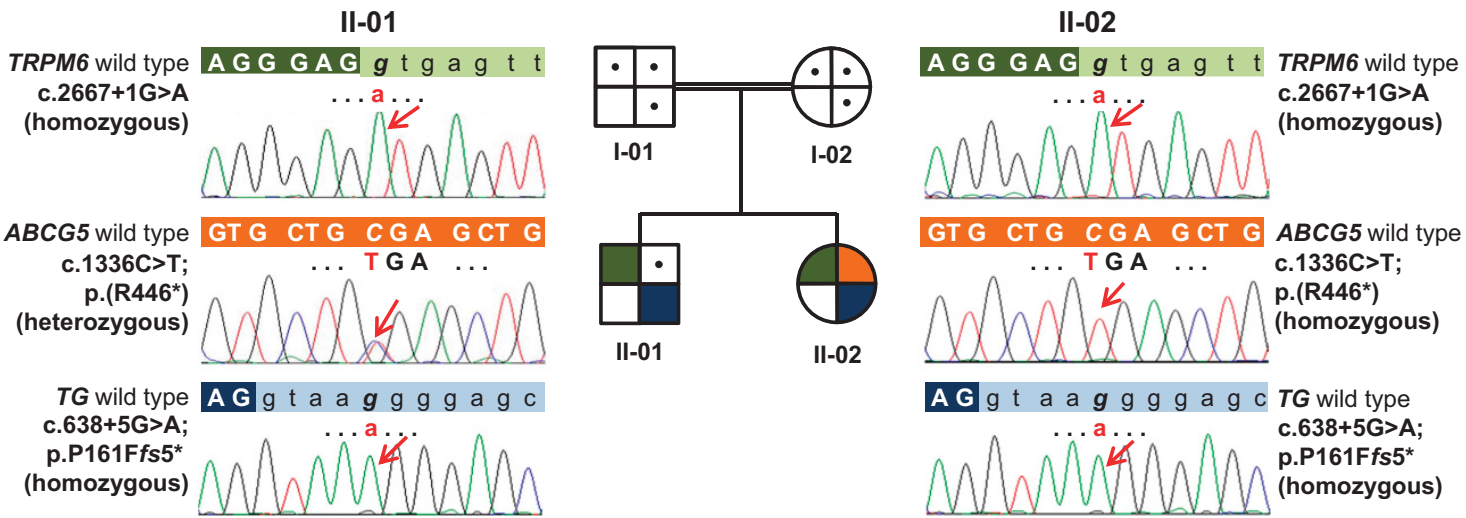

Figure 2 Pedigree of the nuclear family (middle panel), coloring of the filled symbol segments indicates manifestation of phenotypes (green: hypomagnesemia; orange: sitosterolemia; blue: thyroid dyshormonogenesis). Confirmatory genomic Sanger sequencing of TRPM6, ABCG5, and TG mutations in the boy (individual II-01, left panel) and the girl (individual II-02, right panel).

where it mediates magnesium absorption. Mutations in TRPM6 are known to cause autosomal-recessive hypomagnesemia with secondary hypocalcemia (MIM \#602014). ${ }^{11}$ Indeed, the identified variant c.2667 $+1 \mathrm{G}>\mathrm{A}$ has previously been reported in several patients of Turkish origin. ${ }^{12}$ Sanger sequencing in our patients and other family members confirmed heterozygosity of both parents and co-segregation of variant and disease (Figure 2). Hypocalcemia was not regularly observed in either of our patients, but secondary hypocalcemia in patients with TRPM6 mutations is reportedly variable. ${ }^{12}$

\section{A stop variant in ABCG5 causes sitosterolemia}

Among the remaining candidate variants, we identified the c. $1336 \mathrm{C}>\mathrm{T} / \mathrm{p} .\left(\operatorname{Arg} 446^{\star}\right)$ variant, causing a premature stop codon in the ABCG5 gene (Supplementary Table S2). ABCG5 encodes an ATP-binding cassette (ABC) half-transporter expressed in liver, colon, and intestine, which limits intestinal absorption and promotes biliary excretion of sterols. ABCG5 mutations have been identified as a cause of autosomal-recessive sitosterolemia (MIM \#210250). ${ }^{13}$ The p. (Arg446*) variant has been reported previously in a consanguineous family with sitosterolemia from Iran. ${ }^{13}$ Although clinical manifestations of sitosterolemia can be similar to those of familial hypercholesterolemia, the disease can be diagnosed by elevated serum concentrations of the plant sterols, sitosterol and campesterol. We, therefore, measured these sterols in the girl and found them significantly increased, confirming the diagnosis of sitosterolemia (Figure 1c, Table 1). Increased total cholesterol and slightly increased plant sterol concentrations were detected in the heterozygous father. The variant was confirmed by Sanger sequencing and segregated with the phenotype (Figure 2). The girl was started on $10 \mathrm{mg}$ ezetimibe, which after 13 months led to a significant reduction of cholesterol, $5 \alpha$-cholestanol, plant sterols and plant stanols (Figure 1d).

\section{Hypothyroidism caused by a splice variant in TG}

After loosening initial filtering criteria for exon/intron junctions from 2 to $5 \mathrm{bp}$, we identified a variant at a highly conserved +5 splice donor site in intron 5 (IVS5) of the TG gene (c.638+5G>A), encoding thyroglobulin (Supplementary Table S2). The variant was predicted to reduce splicing by a number of splice site prediction programs (Supplementary Figure S3A, Supplementary Table S3). Using a recently published method, ${ }^{14}$ which predicts how strongly a genetic variant affects RNA splicing with very high accuracy, the variant was predicted to lead to a $36.8 \%$ reduction of transcripts containing exon 5 in comparison with the wild-type sequence. For comparison, the corresponding estimate for the known splice variant c. $2667+1 \mathrm{G}>\mathrm{A}$ in TRPM6 was $12.5 \%$. Recessive TG mutations are a known cause of congenital thyroid dyshormonogenesis (MIM \#274700). ${ }^{15}$ TG is a large gene producing many alternatively spliced transcripts. ${ }^{16}$ Mutations affecting splice sites make up a significant portion of all reported pathogenic $T G$ variants. In particular, missplicing of $T G$ premRNA due to another variant in the consensus splice donor site of IVS5 is known to induce congenital goiter, hypothyroidism and subsequent malignant transformation. ${ }^{17}$

We used RT-PCR analysis of patient-derived PBMCs to directly assess the consequences of the novel c.638+5G $>\mathrm{A}$ on $T G$ mRNA. As predicted, the +5 variant resulted in a transcript missing exon 5 (Figures $3 \mathrm{a}$ and $\mathrm{b}$, and Supplementary figure S3B). To exclude that this $T G$ mRNA was a normal transcript splice variant, we performed in silico bioinformatics analyses mapping available RNA-seq data sets to $T G$ exons (Materials and methods section, Supplementary Figure S3C). No natural TG mRNA missing exon 5 was detected either in fetal and adult thyroid tissue or in white blood cells. Different from the known IVS5+1 variant, ${ }^{17}$ semi-quantitative RT-PCR analysis of the intron IVS5 +5 variant indicated that - while overall TG mRNA levels were reduced (Figure 3a, Supplementary Figure S3B) - not all transcripts were mis-spliced in our patients, as evidenced by a faint band of the size of wild-type TG. In keeping with a less penetrant effect of the IVS5+5 variant compared with the known IVS5+1 variant, thyroglobulin in patients' sera was detectable by indirect ELISA (Table 1) and represents a plausible explanation of the mild phenotype of non-goiterous thyroid dyshormonogenesis.

Together, all molecular data confirm that the clinical presentation of this family can be explained by three separate known single-gene disorders.

\section{DISCUSSION}

Here, we report the first instance of a family in whom three separate single-gene disorders, familial hypomagnesemia, sitosterolemia, and thyroid dyshormonogenesis, give rise to a complex and previously unobserved clinical picture. Against our expectations, the molecular cause in offspring from a consanguineous marriage did not reside in a single gene transmitted in an autosomal-recessive fashion. Instead, the blended phenotype resulted from three separate molecular causes, and could only be resolved by their combined identification. These findings have several implications.

First, our findings strengthen the evidence for blended phenotypes ${ }^{4,5}$ arising from more than one underlying monogenic disease. In our 

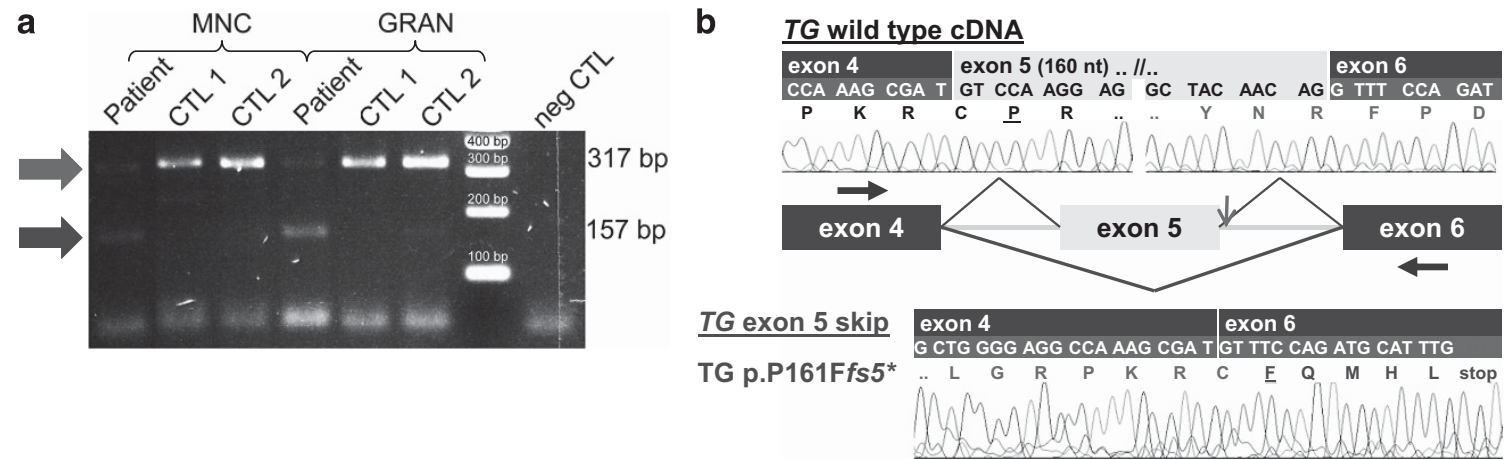

Figure 3 (a) PCR detection of exon 5 skipping in TG transcripts from the girl and healthy controls; cDNA from different leukocyte fractions was amplified with primers annealing in exon 4 (forward) and exon 6 (reverse, blue arrows in b). Expected product sizes are 317 bp in wild-type TG (blue arrow in a) and $157 \mathrm{bp}$ when exon 5 is spliced out (red arrow in a). Note a faint signal at $317 \mathrm{bp}$ in patient 1 , indicating residual normal splicing of TG. (b) Sanger sequencing of exon-spanning cDNA amplicons shown in $\mathbf{b}$. The aberrant splicing results in a frameshift at amino acid 161 (underlined). In the TG reading frame, skipping of exon 5 gives rise to a premature stop codon five codons downstream within exon 6 . The predicted protein from this transcript contains 165 residues, including 161 residues of normal TG sequence. CTL, control; GRAN, granulocytes; MNC, monocytes. A full color version of this figure is available at the European Journal of Human Genetics journal online.

cases, these diseases were independent and the causative variants are located on different chromosomes, which distinguishes this case from blended phenotypes arising from contiguous gene deletion syndromes. Studies of clinical WES and whole-genome sequencing have previously reported on patients with phenotypes arising from two, but not three, independent single-gene disorders. ${ }^{4,5,18-20}$ We propose that a sizeable proportion of the presumed monogenic diseases of yet unknown origin may in fact be due to clustering of several single-gene disorders. One previous large-scale clinical WES study reported that $5 \%$ of patients with a molecular diagnosis had two underlying single-gene disorders. ${ }^{5}$ Another study in patients from consanguineous families reported a slightly higher rate (one out of 18 ) ${ }^{20}$ which may be an underestimate given the identified variants only partially explained the clinical phenotype in two families. It needs to be noted that both estimates were influenced by indication for WES as well as previous knowledge about the identified variants.

In our patients, mutation identification was facilitated by homozygosity mapping as well as previous knowledge about TRPM6, $A B C G 5$, and TG. This task will be more challenging in the setting of de novo mutations, variants of unknown significance or incomplete penetrance, or when each of the causal variants follows a different mode of inheritance. The delineation of a blended phenotype will further be complicated when variants map into genes of yet unknown function. Moreover, even well-characterized variants may provoke unpredictable consequences in a complex genetic background. Variants may, for instance, interact because they directly or indirectly act on a common molecular pathway. In addition, disease genes may have similar functions during embryonic development, and converging developmental effects may also modify the clinical phenotype. Discrete features may thus be ameliorated, aggravated, or completely transformed to a novel phenotype, which - together with epigenetic factors - should contribute to the variable genotype-phenotype relationship commonly observed for single-gene disorders. Together, these issues complicate the estimation of the proportion of presumed monogenic diseases of yet unknown origin that in truth arise from a combination of several monogenic diseases.

Second, our study is the first report of three concomitant independent monogenic diseases in one person, pressing the question for the likelihood of this observation. The prevalence of each of these diseases is exceedingly low, ranging from an estimated 1:100 000 to $1: 1000000$. Therefore, in unrelated individuals and assuming independence of the harmful alleles, the chance of all three of them co-occurring is less than 1 in a quadrillion $\left(10^{15}\right)$. In our case, the patients are offspring from a consanguineous mating and the probability of co-occurrence of the diseases are therefore higher. The number of recessive single-gene diseases observed in offspring of such matings should be influenced by several factors: the number of variants for which the parents are heterozygous, the degree of parental relatedness and reproductive fitness as well as the penetrance and lethality of the resulting recessive diseases. The clustering of singlegene disorders will be facilitated if the underlying variants are hypomorphic or variably penetrant as was the case for $T G$ variant we observed. Yet, the probability of observing three concomitant monogenic recessive diseases in one patient should still be very low, given the probability that a randomly sampled allele at a specific locus is identical-by-descent for first-degree cousins is $6.25 \%$ and considering the factors listed above.

Third, our findings have both practical and theoretical implications for clinical practice. Although both patients continue to receive thyroid hormone replacement therapy and oral magnesium, the lipid-lowering treatment for the girl was switched from cholestyramine to ezetimibe, a specific therapy for sitosterolemia, and put on a diet low in vegetable fat. Ezetimibe blocks the uptake of sterols in the intestine and thereby lowers cholesterol and plant sterol levels. The identification of the underlying ABCG5 variant now allows for monitoring treatment efficacy by measuring blood sterol levels. In addition, the patient will be closely followed for the potential development of cardiovascular disease, although reportedly not all patients with phytosterolemia develop cardiovascular disease. ${ }^{21}$

Advanced diagnostic procedures to investigate exceedingly rare metabolic disorders such as those of sterol absorption are readily available, but their utilization requires a concrete clinical suspicion. As effective prevention and therapy depend on correct classification of pleiotropic phenotypes, circumstantial evidence for the extraordinary is critical. WES has greatly facilitated and accelerated diagnosis in rare disorders. Its cost-effectiveness and utility in clinical diagnosis are increasingly recognized. ${ }^{22,23}$ Even without clinical clues and a working hypothesis, unbiased comprehensive genetic analysis may deliver pathogenic variants. Promoted by success and falling costs, however, widespread use of clinical WES may interfere with clinical judgment. Although WES was instrumental in our case as well, it exemplifies a so far un(der)-recognized pitfall: the successful identification of causative 
variants in one gene may not be sufficient to resolve a clinical phenotype completely, necessitating the search beyond variants that can only account for part of the clinical picture. As the prevalence of blended phenotypes ${ }^{4,5}$ from multiple monogenic disorders is currently unknown, systematic analysis and regular re-analysis of larger WES data sets and, if applicable, integration with results from complementary diagnostic procedures such as karyotyping, is needed to gauge the significance of such 'complex-simplex' diseases in daily practice.

\section{CONFLICT OF INTEREST}

The authors declare no conflict of interest.

\section{ACKNOWLEDGEMENTS}

$\mathrm{YL}$ and AK were funded by the Emmy Noether Programme of the German Research Foundation (KO 3598/2-1). EL and BZ are supported by national and EU-funded Rare Disease Network Programmes FACE (agreement no. 01GM1109A, TP1); SYBIL (FP7 grant agreement no. 602300) and INTERREG IV project A27 to EL. The work of AK and EL was additionally funded through the German Research Foundation (CRC 1140). We thank Anja Kerksiek for excellent technical assistance. We thank the patients and their family members for the participation in this study.

1 Biesecker LG, Green RC: Diagnostic clinical genome and exome sequencing. N Engl J Med 2014; 370: 2418-2425.

2 Boycott KM, Vanstone MR, Bulman DE, MacKenzie AE: Rare-disease genetics in the era of next-generation sequencing: discovery to translation. Nat Rev Genet 2013; 14: 681-691.

3 Bamshad MJ, Ng SB, Bigham AW et al: Exome sequencing as a tool for Mendelian disease gene discovery. Nat Rev Genet 2011; 12: 745-755.

4 Yang Y, Muzny DM, Reid JG et al: Clinical whole-exome sequencing for the diagnosis of mendelian disorders. N Engl J Med 2013; 369: 1502-1511.

5 Yang Y, Muzny DM, Xia F et al: Molecular findings among patients referred for clinical whole-exome sequencing. JAMA 2014; 312: 1870-1879.

6 Sheridan E, Wright J, Small N et al: Risk factors for congenital anomaly in a multiethnic birth cohort: an analysis of the Born in Bradford study. Lancet 2013; 382: 1350-1359.
7 Lausch E, Janecke A, Bros $M$ et al: Genetic deficiency of tartrate-resistant acid phosphatase associated with skeletal dysplasia, cerebral calcifications and autoimmunity. Nat Genet 2011; 43: 132-137.

8 Kamphans T, Krawitz PM: GeneTalk: an expert exchange platform for assessing rare sequence variants in personal genomes. Bioinformatics 2012; 28: 2515-2516.

9 Seelow D, Schuelke M: HomozygosityMapper2012-bridging the gap between homozygosity mapping and deep sequencing. Nucleic Acids Res 2012; 40 W516-W520.

10 Mackay DS, Jones PJ, Myrie SB, Plat J, Lutjohann D: Methodological considerations for the harmonization of non-cholesterol sterol bio-analysis. J Chromatogr B Analyt Technol Biomed Life Sci 2014; 957: 116-122.

11 Schlingmann KP, Weber S, Peters M et al: Hypomagnesemia with secondary hypocalcemia is caused by mutations in TRPM6, a new member of the TRPM gene family. Nat Genet 2002; 31: 166-170.

12 Schlingmann KP, Sassen MC, Weber S et al: Novel TRPM6 mutations in 21 families with primary hypomagnesemia and secondary hypocalcemia. J Am Soc Nephrol 2005; 16: 3061-3069.

13 Mannucci L, Guardamagna O, Bertucci P et al: Beta-sitosterolaemia: a new nonsense mutation in the ABCG5 gene. Eur J Clin Invest 2007; 37: 997-1000.

14 Xiong HY, Alipanahi B, Lee LJ et al: RNA splicing. The human splicing code reveals new insights into the genetic determinants of disease. Science 2015; 347: 1254806.

15 Targovnik HM, Citterio CE, Rivolta CM: Thyroglobulin gene mutations in congenital hypothyroidism. Horm Res Paediatr 2011; 75: 311-321.

16 Targovnik HM, Edouard T, Varela $\mathrm{V}$ et al: Two novel mutations in the thyroglobulin gene as cause of congenital hypothyroidism: identification a cryptic donor splice site in the exon 19. Mol Cell Endocrinol 2012; 348: 313-321.

17 Alzahrani AS, Baitei EY, Zou M, Shi Y: Clinical case seminar: metastatic follicular thyroid carcinoma arising from congenital goiter as a result of a novel splice donor site mutation in the thyroglobulin gene. J Clin Endocrinol Metab 2006; 91: 740-746.

18 Ng SB, Buckingham KJ, Lee $\mathrm{C}$ et al: Exome sequencing identifies the cause of a mendelian disorder. Nat Genet 2010; 42: 30-35.

19 Roach JC, Glusman G, Smit AF et al: Analysis of genetic inheritance in a family quartet by whole-genome sequencing. Science 2010; 328: 636-639.

20 Makrythanasis P, Nelis M, Santoni FA et al: Diagnostic exome sequencing to elucidate the genetic basis of likely recessive disorders in consanguineous families. Hum Mutat 2014; 35: 1203-1210.

21 Hansel B, Carrie A, Brun-Druc $\mathrm{N}$ et al: Premature atherosclerosis is not systematic in phytosterolemic patients: severe hypercholesterolemia as a confounding factor in five subjects. Atherosclerosis 2014; 234: 162-168.

22 Valencia CA, Husami A, Holle J et al: Clinical impact and cost-effectiveness of whole exome sequencing as a diagnostic tool: a pediatric center's experience. Front Pediatr 2015; 3: 67.

23 Sawyer SL, Hartley T, Dyment DA et al: Utility of whole-exome sequencing for those near the end of the diagnostic odyssey: time to address gaps in care. Clin Genet 2015; e-pub ahead of print 18 August 2015; doi:10.1111/cge.12654.

Supplementary Information accompanies this paper on European Journal of Human Genetics website (http://www.nature.com/ejhg) 